\title{
Long-term Escitalopram Treatment in Korean Patients with Panic Disorder: A Prospective, Naturalistic, Open-label, Multicenter Trial
}

\author{
Kwan-Woo Choi ${ }^{1}$, Jong-Min Woo ${ }^{2}$, Youl-Ri Kim², Seung-Hwan Lee ${ }^{3}$, Sang-Yeol Lee ${ }^{4}$, Eui-Jung Kim ${ }^{5}$, \\ Sang-Keun Chung ${ }^{6}$, Eun-Ho Kang ${ }^{1}$, Jae-Hon Lee', Bum-Hee $\mathrm{Yu}^{1}$ \\ ${ }^{1}$ Department of Psychiatry, Samsung Medical Center, Sungkyunkwan University School of Medicine, Seoul, ${ }^{2}$ Department of Neuropsychiatry \\ and Stress Research Institute, Seoul Paik Hospital, Inje University School of Medicine, Seoul, ${ }^{3}$ Department of Neuropsychiatry, Ilsan Paik \\ Hospital, Inje University School of Medicine, Goyang, ${ }^{4}$ Department of Psychiatry, Wonkwang University School of Medicine, Iksan, \\ ${ }^{5}$ Department of Psychiatry, Ewha Womans University College of Medicine, Seoul, ${ }^{6}$ Department of Psychiatry, Chonbuk National University \\ Medical School, Jeonju, Korea
}

\begin{abstract}
Objective: Panic disorder is characterized by recurrent panic attacks, persistent concerns about additional attacks, and worry about the implications of the attack or significant changes in behavior related to the attacks. We examined the efficacy of 24-week naturalistic, open-label escitalopram treatment in terms of the response and remission rates and functional disability in 119 adult Korean patients with panic disorder from 6 clinical centers in South Korea.

Methods: Clinical severity and functional impairment were assessed at baseline and at 4, 12, and 24 weeks after the treatment using the Panic Disorder Severity Scale and Sheehan Disability Scale. Ninety-six patients (80.7\%) showed a treatment response, and 87 patients $(73.1 \%)$ had attained remission after 24 weeks of escitalopram treatment.

Results: Continuous improvement in the Panic Disorder Severity Scale and Sheehan Disability Scale scores was found over the 24 weeks of treatment.

Conclusion: These findings suggest that escitalopram treatment is very effective for panic disorder in terms of both response and remission rates and that long-term pharmacotherapy with escitalopram continuously improved panic symptoms and functional disability in Korean patients with panic disorder.
\end{abstract}

KEY WORDS: Panic disorder; Escitalopram; Anxiety, psychopharmacology; Antidepressants.

\section{INTRODUCTION}

Panic disorder (PD) is characterized by recurrent panic attacks, persistent concerns about additional attacks, and worry about the implications of the attacks or significant changes in behavior related to the attacks. In the US, PD is estimated to occur in approximately $3.5 \%$ of the general population and up to $20 \%$ of primary care patients. ${ }^{1,2)}$ This relatively common syndrome is chronic and has an adverse effect on patients' quality of life. ${ }^{3,4)}$ Recent studies have evaluated how PD affects quality of life due to its adverse impact on vocational, family, and social functioning. ${ }^{5-8)}$ Ormel et al. ${ }^{9)}$ found that more than half of PD

Received: November 10, 2011 / Revised: January 16, 2012 Accepted: January 30, 2012

Address for correspondence: Bum-Hee $\mathrm{Yu}, \mathrm{MD}, \mathrm{PhD}$ Department of Psychiatry, Samsung Medical Center, Sungkyunkwan University School of Medicine, Irwon-dong, Gangnam-gu, Seoul 135-710, Korea

Tel: +82-2-3410-3583, Fax: +82-2-3410-6957

E-mail: bhyu@skku.edu patients reported moderate or severe occupational role dysfunction and physical disability. Hence, an improvement in the functional disability should be considered as important as an improvement in panic symptom profiles when evaluating the efficacy of PD treatment.

Currently, selective serotonin-reuptake inhibitors (SSRIs) are indicated as the first-line treatment for PD. SSRIs are relatively safe and have few side effects. ${ }^{10}$ ) Escitalopram is the most recently developed SSRI and is used widely for the treatment of patients with PD. To our knowledge, however, only two studies have examined escitalopram for the treatment of $\mathrm{PD}$, and these only showed the efficacy of escitalopram for 8 and 10 weeks. ${ }^{11,12)}$ Stahl et $a{ }^{11)}$ conducted a randomized, double-blind, placebocontrolled, 10-week trial with 377 PD patients. In that study, escitalopram and citalopram were more effective than a placebo in reducing both anticipatory anxiety and the intensity of the panic attack. Rampello et al. ${ }^{12)}$ conducted an open-label, naturalistic study that showed the efficacy of escitalopram in subjects aged 65 or older after 
8 weeks. However, these two studies had some limitations: both were short-term studies and could not reflect the natural clinical course of PD because a substantial number of PD patients suffer from residual symptoms, such as anticipatory anxiety, even after standard treatment. ${ }^{13)}$ Additionally, the study by Rampello et al. ${ }^{12)}$ included only elderly patients, so their results cannot be extended to a general PD population. Therefore, a long- term study in the general PD population is needed to examine the real anti-panic efficacy of escitalopram.

Consequently, we examined the efficacy of 24-week prospective, naturalistic, open-labeled escitalopram treatment in terms of panic symptoms, functional disability, and quality of life in PD patients.

\section{METHODS}

This was a prospective, naturalistic, open-label, multicenter trial of escitalopram in Korean PD patients with or without agoraphobia from 6 clinical centers in South Korea.

\section{Subjects}

Experienced psychiatrists administered the structured clinical interview for the Diagnostic and Statistical Manual of Mental Disorders, Fourth Edition (SCID-IV) to all subjects, and we recruited study subjects who were diagnosed with PD with or without agoraphobia from 2007 to 2009.

Exclusion criteria consisted of the presence of serious or unstable medical illnesses, high suicide risk, or major mental illnesses such as major depressive disorder, bipolar affective disorder, schizophrenia, other psychotic disorders, organic brain syndrome, and alcohol or other substance use disorders. We also excluded pregnant or lactating women and women with childbearing potential who were not using medically accepted contraception.

We ultimately enrolled 119 PD patients (66 males, 53 females). The Institutional Review Board of Samsung Medical Center approved this study, and all patients gave informed consent before participating in the study.

\section{Assessment}

The primary outcome measures were the remission rate and response rate of PD. Secondary outcome measures included changes from baseline in the Sheehan Disability Scale (SDS) ${ }^{14)}$ and the Panic Disorder Severity Scale (PDSS). ${ }^{15)}$ The PDSS is a 7-item scale used to assess multiple dimensions of PD severity. Each item is rated from 0 to 4 , so total score ranges from 0 to 28 . The SDS consists of three Likert (0 to 10) items measuring impairment in work, social relationships, and responsibilities at home and with family. We defined clinical remission as the absence of full panic attacks and a PDSS score $\leq 7$, which is in line with the suggestion made by Furukawa et al. ${ }^{16)} \mathrm{We}$ defined clinical response as a more than $50 \%$ reduction in the PDSS score. All patients were assessed using the Hamilton Rating Scale for Depression, PDSS, and SDS at baseline and at 4, 12, and 24 weeks after beginning treatment.

\section{Treatment}

Before the treatment with escitalopram, all participants had a 2-week drug-free or washout period (a 5-week washout period for fluoxetine). All patients were treated with escitalopram at an initial dose of 5 or $10 \mathrm{mg}$ per day, and the dose was increased up to $20 \mathrm{mg}$ per day according to the clinicians' judgment. The mean daily dose ( \pm standard deviation) of escitalopram was $11.65 \pm 3.83 \mathrm{mg} /$ day during the study period. During the first 4 weeks, the use of alprazolam or clonazepam was allowed, but it was tapered off until 12 weeks after treatment. Zolpidem was allowed for controlling insomnia.

\section{Statistical Analysis}

Intention-to-treat analysis was performed using the last observation carried forward (LOCF) method, and repeated-measures ANOVA was used to test for improvement in the PDSS and SDS scores. The Bonferroni correction for multiple comparisons was used. SPSS ver. 17.0 (SPSS Inc., Chicago, IL, USA)was used for all statistical analyses, and statistical significance was defined at the 0.05 level (two-tailed tests).

\section{RESULTS}

\section{Demographic and Clinical Characteristics}

The demographic and clinical characteristics of the patients at baseline $(n=119)$ are summarized in Table 1. At baseline, the age of the subjects ranged from 20 to 70 years (mean $=41.68$ years), and $53(45.5 \%)$ were female. Fortysix PD patients (38.7\%) had agoraphobia. Fifty-seven patients $(47.9 \%)$ completed 24 weeks of treatment, and no significant demographic or clinical differences were found between study completers and those who dropped out of the study. 
Table 1. Demographic and clinical characteristics of subjects at baseline

\begin{tabular}{lc}
\hline & ITT $(n=119$, mean \pm SD $)$ \\
\hline Age (year) & $41.68 \pm 9.60$ \\
Sex (M/F) & $66 / 53$ \\
Agoraphobia (with/without) & $46 / 73$ \\
HAM-D 17 & $12.45 \pm 4.27$ \\
PDSS & $15.75 \pm 4.38$ \\
SDS total & $16.30 \pm 7.12$ \\
SDS work & $5.95 \pm 2.72$ \\
SDS relationship & $5.23 \pm 2.63$ \\
SDS home & $5.13 \pm 2.59$ \\
Escitalopram dose (mg) & $8.47 \pm 3.71$ \\
\hline
\end{tabular}

HAM-D, Hamilton Rating Scale for Depression; PDSS, Panic Disorder Severity Scale; SDS, Sheehan Disability Scale; ITT, intertion-to-treat; $\mathrm{SD}$, standard deviation.

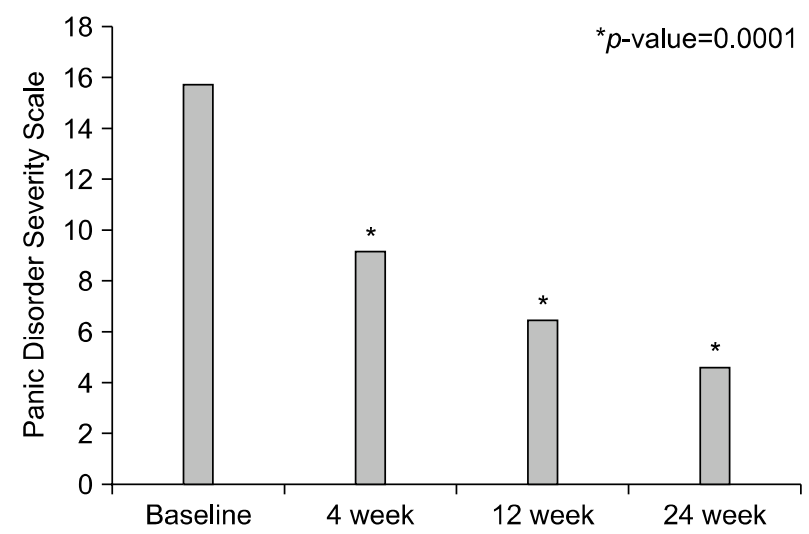

Fig. 1. Changes of the PDSS total score over time.

\section{PDSS}

Among the 119 PD patients, 87 patients (73.1\%) had attained remission by the end of the treatment. The response rate was $45.4,69.7$, and $80.7 \%$ at weeks 4,12 , and 24 , respectively.

The total PDSS scores over time are shown in Fig. 1. At the LOCF week 24 evaluation, significant improvement in the total PDSS score was observed $(-11.16 \pm 6.51, p=$ $0.0001 ; 95 \%$ confidence interval -12.76 to -9.56$)$. In the post hoc analysis, we found a continuous significant improvement from baseline to weeks 4,12 , and 24 ( $p=$ $0.0001 ; F=115.65 ; \mathrm{d} f=3)$.

\section{SDS}

The SDS score over time is shown in Fig. 2. We found significant improvements in three areas of the SDS, i.e., at work, in social relationships, and in responsibilities at home and with family, at baseline and at weeks 4,12 , and $24(p=0.0001)$.

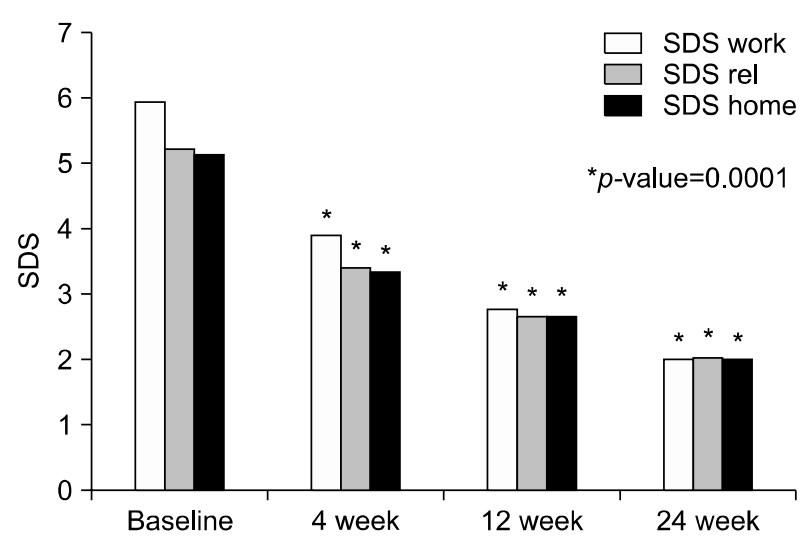

Fig. 2. Score changes of the SDS 3 domains over time. SDS, Sheehan Disability Scale.

\section{DISCUSSION}

Our study demonstrated the long-term efficacy of escitalopram in PD patients. Of 119 study subjects, $73.1 \%$ attained remission by the end of the study. We also found that the response rate of $\mathrm{PD}$ patients treated with escitalopram was $80.7 \%$ at week 24 . After the 24 -week treatment with escitalopram, a significant improvement in the total PDSS score was observed beginning from week 4, and the improvement continued progressively until the end of the study. Additionally, we found a continuous significant improvement in the SDS sub-domains (work, social relationships, and responsibilities at home and with family) at baseline and weeks 4, 12, and 24.

Consistent with the two previous escitalopram studies, ${ }^{11,12)}$ our study demonstrated that treatment with escitalopram was effective, even at an early stage of treatment; $45.4 \%$ of the PD patients showed a treatment response to escitalopram at week 4. Furthermore, $69.7 \%$ of them showed a treatment response to escitalopram at week 12, which was compatible to a recent PD study using venlafaxine. ${ }^{17)}$ Liebowitz et al. ${ }^{17)}$ found that treatment with venlafaxine-extended release had a $74 \%$ response rate (they defined a response as a $40 \%$ reduction in the PDSS) in their 10-week, double-blind, placebo-controlled study of PD patients, although their definition of treatment response was somewhat different from ours.

The response rate in our study increased continuously until the end of the study $(45.4,69.7$, and $80.6 \%$ at weeks 4,12 , and 24 , respectively). To our knowledge, very few studies have evaluated the efficacy of SSRI or serotonin-norepinephrine reuptake inhibitor treatment for more than 12 weeks. Therefore, these results suggest that long-term treatment with escitalopram is necessary to ob- 
tain the best treatment response in terms of the panic symptoms and functional disabilities of PD.

The high remission rate $(73.1 \%)$ in this study was generally consistent with that of previous short-term pharmacotherapy trials in PD. Previous studies showed the efficacy of antidepressants using the definition of remission as a panic-free state at the end point of the study. ${ }^{18)}$ In our study, 96 of $119(80.7 \%)$ patients had a panic-free state at week 24, which was compatible to previous studies using paroxetine $40 \mathrm{mg} /$ day $^{19)}(86.0 \%$ remission rate at week $10)$ and venlafaxine $225 \mathrm{mg} /$ day $^{20)}(70 \%$ remission rate at week 10).

PD patients suffer from significant functional impairment. The analysis of the Medical Outcome Study showed that PD patients had high levels of psychological distress and marked limitations in role functioning compared with patients who had hypertension, diabetes, heart disease, or arthritis. ${ }^{19,21)}$ In our study, the PD patients showed a significant improvement in three domains of the SDS (work, social relationships, and responsibilities at home and with family), which was compatible to the result of a previous study using fluvoxamine as anti-panic medication. ${ }^{22)}$ Therefore, escitalopram appears to be effective in enhancing work, social, and family functioning and in improving panic symptoms in PD patients.

The current American Psychiatric Association practice guidelines for the treatment of PD recommend continuing SSRI pharmacotherapy for at least 1 year. Our study suggests that escitalopram treatment should be maintained for more than 6 months in PD patients to obtain the best treatment outcome, which supports the current APA practice guidelines for the treatment of PD.

In conclusion, these findings suggest that escitalopram is very effective for the treatment of PD. Long-term pharmacotherapy with escitalopram improves panic symptoms and functional disabilities continuously in Korean patients with PD.

\section{Acknowledgments}

The study was given financial support in the form of an unrestricted grant from $\mathrm{H}$. Lundbeck $\mathrm{A} / \mathrm{S}$, who was not involved in the creation of the study protocol, data analysis, data interpretation, or writing of the manuscript. This study was also partly supported by Clinical Research Development Program grants (CRS 107-18-1 and 107-48-2) from the Samsung Biomedical Research Institute.

\section{REFERENCES}

1. Kessler RC, Chiu WT, Jin R, Ruscio AM, Shear K, Walters EE. The epidemiology of panic attacks, panic disorder, and agoraphobia in the National Comorbidity Survey Replication. Arch Gen Psychiatry 2006;63:415-424.

2. Kroenke K, Spitzer RL, Williams JB, Monahan PO, Löwe B. Anxiety disorders in primary care: prevalence, impairment, comorbidity, and detection. Ann Intern Med 2007; 146:317-325.

3. Noyes R Jr, Reich J, Christiansen J, Suelzer M, Pfohl B, Coryell WA. Outcome of panic disorder. Relationship to diagnostic subtypes and comorbidity. Arch Gen Psychiatry 1990;47:809-818.

4. Wittchen HU, Essau CA. Epidemiology of panic disorder: progress and unresolved issues. J Psychiatr Res 1993;27 Suppl 1:47-68.

5. Cramer V, Torgersen S, Kringlen E. Quality of life and anxiety disorders: a population study. J Nerv Ment Dis 2005;193:196-202.

6. Bandelow B, Stein DJ, Dolberg OT, Andersen HF, Baldwin DS. Improvement of quality of life in panic disorder with escitalopram, citalopram, or placebo. Pharmacopsychiatry 2007;40:152-156.

7. Markowitz JS, Weissman MM, Ouellette R, Lish JD, Klerman GL. Quality of life in panic disorder. Arch Gen Psychiatry 1989;46:984-992.

8. Weissman MM. Panic disorder: impact on quality of life. J Clin Psychiatry 1991;52(Suppl):6-8.

9. Ormel J, VonKorff M, Ustun TB, Pini S, Korten A, Oldehinkel T. Common mental disorders and disability across cultures. Results from the WHO Collaborative Study on Psychological Problems in General Health Care. JAMA 1994;272:1741-1748.

10. Shear MK, Maser JD. Standardized assessment for panic disorder research. A conference report. Arch Gen Psychiatry 1994;51:346-354.

11. Stahl SM, Gergel I, Li D. Escitalopram in the treatment of panic disorder: a randomized, double-blind, placebo-controlled trial. J Clin Psychiatry 2003;64:1322-1327.

12. Rampello L, Alvano A, Raffaele R, Malaguarnera M, Vecchio I. New possibilities of treatment for panic attacks in elderly patients: escitalopram versus citalopram. J Clin Psychopharmacol 2006;26:67-70.

13. Bandelow B, Rüther E. Treatment-resistant panic disorder. CNS Spectr 2004;9:725-739.

14. Sheehan DV. The Anxiety Disease. New York: Scribner's; 1983.

15. Shear MK, Clark D, Feske U. The road to recovery in panic disorder: response, remission, and relapse. J Clin Psychiatry 1998;59(Suppl 8):4-8.

16. Furukawa TA, Katherine Shear M, Barlow DH, Gorman JM, Woods SW, Money R, et al. Evidence-based guidelines for interpretation of the Panic Disorder Severity Scale. Depress Anxiety 2009;26:922-929.

17. Liebowitz MR, Asnis G, Mangano R, Tzanis E. A double-blind, placebo-controlled, parallel-group, flexibledose study of venlafaxine extended release capsules in adult outpatients with panic disorder. J Clin Psychiatry 2009; 70:550-561.

18. Mochcovitch MD, Nardi AE. Selective serotonin-reuptake inhibitors in the treatment of panic disorder: a systematic review of placebo-controlled studies. Expert Rev Neurother 2010;10:1285-1293.

19. Ballenger JC, Wheadon DE, Steiner M, Bushnell W, Gergel 
IP. Double-blind, fixed-dose, placebo-controlled study of paroxetine in the treatment of panic disorder. Am J Psychiatry 1998;155:36-42.

20. Pollack M, Mangano R, Entsuah R, Tzanis E, Simon NM, Zhang Y. A randomized controlled trial of venlafaxine $E R$ and paroxetine in the treatment of outpatients with panic disorder. Psychopharmacology (Berl) 2007;194:233-242.
21. Sherbourne CD, Wells KB, Judd LL. Functioning and well-being of patients with panic disorder. Am J Psychiatry 1996;153:213-218.

22. Asnis GM, Hameedi FA, Goddard AW, Potkin SG, Black $\mathrm{D}$, Jameel $\mathrm{M}$, et al. Fluvoxamine in the treatment of panic disorder: a multi-center, double-blind, placebo-controlled study in outpatients. Psychiatry Res 2001;103:1-14. 